\title{
$\mathrm{R} C \& \mathrm{C}$
}

REVISTA DE CONTABILIDADE E CONTROLADORIA

\section{AVALIAÇÃO DE DESEMPENHO ORGANIZACIONAL EM PEQUENAS E MÉDIAS EMPRESAS: ESTUDO MULTICASO EM CONCESSIONÁRIAS DE MÁQUINAS E IMPLEMENTOS AGRÍCOLAS}

\section{EVALUATION OF ORGANIZATIONAL PERFORMANCE IN SMALL AND MEDIUM ENTERPRISES: MULTICASE STUDY IN DEALERS AGRICULTURAL MACHINERY AND IMPLEMENTS}

Recebido em 09.06.2014 | Aceite final em 04.09.2015 | Nota: este artigo foi aceito pelo Editor Jorge Eduardo Scarpin e passou por uma avaliação double blind review A reprodução dos artigos, total ou parcial, pode ser feita desde que citada a fonte.

\begin{abstract}
THALYSON RENAN BITENCOURT MACHADO Mestrando em Ciências Contábeis | Universidade Federal de Uberlândia | Especialista em Controladoria Empresarial | Universidade Estácio de Sá | Professor da Faculdade Católica do Tocantins | Av. Teotônio Segurado, Quadra 1402 Sul, Lote 01, Plano Diretor Sul, Palmas-TO| Telefone (63) 8449-6366 | e-mail: thalyson.renan@outlook.com
\end{abstract}

GEOVANE PAULO SORNBERGER Doutorando em Administração | Universidade do Vale do Rio dos Sinos | Mestre em Engenharia de Produção | Universidade Federal de São Carlos | Professor da Universidade do Estado de Mato Grosso | Campus Universitário de Sinop | Av. dos Ingás, 3001, Jardim Imperial, Sinop-MT | Telefone (66) 9687-4427 | e-mail: gps@unemat.br

\section{FERNANDA MOSSELINE JOSENDE COAN} Mestre em Ciências Sociais | Universidade do Vale do Rio dos Sinos | Professora da Universidade do Estado de Mato Grosso | Campus Universitário de Sinop | Av. dos Ingás, 3001, Jardim Imperial, Sinop-MT | Telefone (66) 9985-1879 | e-mail: fernanda@unemat-net.br

\section{RESUMO}

Este trabalho tem por objetivo analisar o processo de avaliação de desempenho realizado em pequenas e médias empresas, especificamente em concessionárias de máquinas e implementos agrícolas, com base nos elementos-chave apresentados por Waggoner, Neely e Kennerley (1999). O estudo foi realizado por meio de entrevistas aos gestores das referidas organizações, cujos dados foram analisados estatisticamente, com posterior realização de um teste de hipóteses. Embora os gestores afirmem que não 
implementaram nenhum modelo de avaliação de desempenho abordado na literatura, em razão do desconhecimento da existência das ferramentas e/ou dos benefícios gerados, constatou-se que o processo realizado nessas organizações contempla os elementos-chave de Waggoner, Neely e Kennerley (1999). Os resultados demonstram que a avaliação de desempenho é útil e necessária também às pequenas e médias empresas, assegurando o cumprimento do planejamento organizacional, subsidiando o controle gerencial, possibilitando a arguição da relação de causa e efeito do desempenho organizacional, fornecendo informações úteis e tempestivas, facilitando a análise crítica do desempenho global da empresa e permitindo estabelecer uma relação entre o desempenho passado, presente e esperado para futuro da organização. Quanto aos indicadores utilizados, constatou-se que as organizações utilizam indicadores financeiros e não financeiros, como rentabilidade, liquidez, faturamento, participação no mercado e ações socioambientais. Conclui-se que avaliação de desempenho é útil e necessária às empresas, independentemente do seu porte, pois asseguram o planejamento organizacional, suprem necessidades gerenciais e auxilia no processo de tomada de decisões.

Palavras-Chave: Avaliação de Desempenho Organizacional. Mensuração de Resultados. Pequenas e Médias Empresas.

\begin{abstract}
This work aims to analyze the performance assessment process carried at small and medium companies, focusing on dealers of agricultural machinery and implements, based on the key elements presented by Waggoner, Neel and Kennedy (1999). The study was conducted by interviewing managers of those organizations whose data were statistically analyzed and subsequent realization of a hypothesis test. Although the managers affirm they haven't made use of any assessment of performance approached by the literature, once they are not familiar with the existence of such tools and its benefits, we could notice that the process carried out in these organizations includes the key elements of Waggoner, Neely and Kennerley (1999).The results show that the performance evaluation is useful and necessary also to small and medium enterprises, ensuring compliance of organizational planning, supporting the management control, allowing the complaint of the cause and effect of organizational performance by providing useful and timely information. It provides a critical analysis of the overall performance of the company at the same time enable us to relate past performance, present and expected future for the organization. About the indicators used, it was found that organizations use financial and non-financial indicators such as profitability, liquidity, sales, market share and environmental actions. We conclude that performance evaluation is useful and necessary to enterprises, regardless of their size, ensure organizational planning, supply management needs and assists in decision-making.

Keywords: Organizational Performance Assessment. Results Measurements. Small and Medium Companies.
\end{abstract}




\section{INTRODUÇÃO}

O desenvolvimento empresarial incita a demanda por informações cada vez mais úteis e tempestivas, fazendo com que a contabilidade assuma um relevante papel no monitoramento do desempenho organizacional, sendo o modelo de gestão a principal ferramenta responsável por suportar e disponibilizar informações que auxiliem no processo gerencial, materializando-se com o planejamento, a execução e o controle das atividades a fim de fazer com que a empresa consiga obter os resultados esperados, cabendo à contabilidade, enquanto instrumentos de evidenciação da situação econômico-financeira e patrimonial, fornecer informações sobre o desempenho organizacional (FREZATTI et al., 2009).

A demanda por informações resultou na criação de ferramentas de suporte à gestão, como por exemplo, os sistemas de avaliação de desempenho organizacional (ADO), cuja função é fornecer informações acerca dos resultados alcançados em determinado período, em função do cumprimento ou não do planejamento (SANTOS, 2010), sendo, portanto, uma ferramenta imprescindível para o gerenciamento de um complexo conjunto de informações relevantes ao desenvolvimento e à manutenção das atividades empresariais (BORTOLUZZI et al., 2010). No entanto, os sistemas de avaliação de desempenho foram criados para grandes organizações, não havendo sistemas especificamente criados para pequenas e médias empresas pelo fato de serem pouco adotados, em razão do baixo volume de recursos humanos e financeiros e do desconhecimento dos benefícios gerados (GARENGO et al., 2005).

Nas últimas décadas, inúmeras organizações de pequeno e médio porte buscaram ferramentas de suporte ao processo de mensuração e avaliação de resultados; no entanto, como essas ferramentas têm foco em grandes organizações, não suprem as necessidades das pequenas e médias empresas (BORTOLUZZI et al., 2010). Todavia, as pequenas e médias empresas, cada vez mais em ascensão, necessitam de ferramentas úteis e tempestivas que suportem suas práticas gerenciais de modo a auxiliar seus gestores no processo de tomada de decisão, em especial naquelas ligadas ao setor do agronegócio, em razão da expansão da produção agrícola nacional e dos segmentos direta e indiretamente interligados ao setor, principalmente em relação ao mercado de máquinas e implementos agrícolas (IBGE, 2012).

Nesse contexto, considerando-se que o processo de avaliação de desempenho tem por foco grandes organizações, e diante da importância de tal processo também para as empresas de pequeno e médio porte, especialmente aquelas ligadas ao setor do agronegócio, buscou-se responder a seguinte questão-problema: como é o processo de avaliação de desempenho organizacional em pequenas e médias concessionárias de máquinas e implementos agrícolas? Este estudo tem como principal objetivo analisar o processo de avaliação de desempenho realizado em concessionárias de máquinas e implementos agrícolas. Com o propósito de cumprir o objetivo geral foram definidos os seguintes objetivos secundários: i) examinar se essas empresas realizam o processo de avaliação de desempenho organizacional; ii) identificar se implementaram ou não algum modelo de avaliação de desempenho abordado na literatura ; e iii) identificar os indicadores e os aspectos considerados e caracterizar tal processo.

A presente pesquisa justifica-se por estudar o processo de avaliação de desempenho realizado em pequenas e médias empresas, especialmente em concessionárias de máquinas e implementos agrícolas, e por analisar uma abordagem menosprezada em relação às tais organizações, possibilitando a caracterização de tal processo nestas organizações.

A estrutura deste artigo é composta por seis títulos, iniciando-se com a introdução. Em seguida aborda a avaliação de desempenho organizacional, seus conceitos e objetivos, sua relação com o controle gerencial. Na sequência discorre sobre a avaliação de desempenho em pequenas e médias 
empresas, sobre os sistemas de avaliação de desempenho e sobre os indicadores de desempenho. Ao final, apresenta os procedimentos metodológicos, a descrição e análise dos dados, a conclusão e as bibliografias utilizadas na presente pesquisa.

\section{FUNDAMENTAÇÃO TEÓRICA}

\subsection{AVALIAÇÃO DE DESEMPENHO ORGANIZACIONAL}

Constituir e manter uma organização que consiga perdurar por um longo período de tempo é o que todo empresário almeja, idealizando-a de forma a mantê-la ativa e competitiva para colher os frutos de seu investimento; entretanto, esse processo não é uma tarefa fácil e nem tampouco simples, pois a constituição, a manutenção e a gestão de uma organização formam um complexo conjunto de funções que muito exige dos gestores e outros interessados em seu desenvolvimento econômico, financeiro, social e ambiental (FREZATTI et al., 2009).

Segundo Müller (2003), a manutenção da competitividade empresarial está relacionada ao modo pelo qual a organização se projeta para alcançar seus objetivos e cumprir suas metas, alinhadas, sobretudo, com a estratégia escolhida. No entanto, a implementação de um bom planejamento estratégico não é garantia de sucesso empresarial, sendo necessário também que seja implementado um processo de mensuração e avaliação de resultados (BORTOLUZZI et al., 2010), geralmente realizado por meio de sistemas de avaliação de desempenho, o qual Simons (2000) apud Corrêa e Júnior (2008, p. 53) define como "sistema de informações que os administradores usam para rastrear a implementação da estratégia do negócio, comparando-se os resultados reais aos objetivos e metas estratégicas".

Na concepção de Neely et al. (2000), um sistema de avaliação de desempenho é compreendido como a integração dos processos de coleta de dados (input), tratamento, análise, interpretação e disseminação dos indicadores (output) que norteiam o processo organizacional. A expressão avaliação de desempenho, entretanto, possui sentido amplo, oscilando conforme o emprego do termo desempenho e do contexto relativo à avaliação. A compreensão do processo de avaliação de desempenho requer a preliminar compreensão desses conceitos, os quais referem-se, respectivamente: i) à apuração dos resultados auferidos em determinado período que podem ser mensurados; e ii) à análise de determinada situação, julgando se o planejamento foi ou não cumprido com a atribuição de valor (SANTOS, 2010).

Nesse sentido, o processo de avaliação de desempenho pode ser compreendido como o ato ou efeito de julgar ou atribuir valor a determinado resultado em consonância com as expectativas preestabelecidas (SILVA; NIYAMA, 2011), concretizando-se com a mensuração, compreendida como a quantificação da eficácia e da eficiência das atividades empresariais por meio de um indicador, o qual é a métrica utilizada nessa quantificação. Dessa forma, os indicadores utilizados para quantificar a eficiência e a eficácia dessas atividades formam um sistema de medição de desempenho (NEELY et al., 2000). Tem-se, portanto, que a avaliação de desempenho é o processo que permite que uma organização mensure seus resultados e a avalie se cumpriu ou não seus objetivos, caracterizando-se como um instrumento organizacional necessário à tomada de decisões.

\subsection{O CONTROLE GERENCIAL E O PROCESSO DE AVALIAÇÃO DE DESEMPENHO}

Segundo Müller (2003, p. 103) a avaliação de desempenho "encontra-se incorporada nas funções administrativas de controle operacional e planejamento estratégico [...], o planejamento estratégico 
deve, portanto, incluir uma abordagem estruturada para executar sua monitoração e medição". Na concepção de Schmidt et al. (2006, p. 37)

\begin{abstract}
as empresas mais preparadas para enfrentar o ambiente competitivo atual são as que possuem um sistema integrado e harmônico de gerenciamento apoiado na formulação de estratégias, no planejamento de execução das estratégias, na medição do desempenho em todas as fases da implementação da estratégia, com um sistema de controle suficientemente eficiente para detectar e analisar rapidamente os desvios para, em seguida, retroalimentar o sistema para corrigir os rumos.
\end{abstract}

A efetivação do processo de avaliação de desempenho como ferramenta de auxílio à gestão empresarial depende de um eficiente sistema de controle gerencial, pois somente pode ser medido e avaliado aquilo que é gerenciado (KAPLAN; NORTON, 1997 apud DUTRA, 2005). No entanto, há diferentes métodos de controle gerencial em razão dos diferentes modelos de gestão implementados, apresentando diferenças de uma organização para outra; no entanto, embora haja algumas oscilações, o ciclo do processo de controle gerencial quando completo compreende no mínimo quatro fases distintas: "(i) estabelecimento de padrões; (ii) comparação do desempenho real com o esperado; (iii) identificação de eventuais desvios; e (iv) ações corretivas, quando necessário" (FREZATTI et al., 2009, p. 156). Nota-se, portanto, a importância do planejamento gerencial conjecturado no modelo administrativo organizacional, bem como, a importância da implementação de um sistema de avaliação de desempenho como auxílio ao planejamento estratégico empresarial.

\title{
2.2.1 AVALIAÇÃO DE DESEMPENHO ORGANIZACIONAL EM PEQUENAS E MÉDIAS EMPRESAS
}

O porte empresarial exerce forte influência na estrutura organizacional, determinando características intrínsecas que resultam em estruturas administrativas específicas, problemas distintos e deficiências que influenciam no modelo de gestão e atuação de cada organização. Em relação às pequenas e médias empresas, as principais características referem-se à estrutura organizacional informal, ao baixo nível de especialização, à pouca departamentalização e à gestão centralizada (MORAES et al., 2007). Souza (2011) afirma que essas empresas são importantes para um mercado altamente flexível, pois tendem a inovar em nichos específicos, ocupando lugares de destaque na geração de renda com grande absorção de mão de obra.

Entretanto, em termos de capacidade organizacional, essas organizações enfrentam problemas que limitam ou impedem seu crescimento, necessitando de um processo de gestão diferenciado. As pequenas e médias empresas encontram-se em um mercado altamente competitivo, no qual necessitam de ferramentas que auxiliem no aprimoramento do modelo de gestão implementado, porém muitas vezes encontram-se despreparadas para enfrentar os desafios impostos (LEONE, 1999). Embora não haja sistemas de avaliação de desempenho especificamente criados para empresas deste porte, há razões suficientes que justificam a criação de sistemas para essas organizações, como o ambiente competitivo e organizacional e as práticas gerenciais, intrínsecos a este tipo de empresa (GARENGO et al., 2005).

Em razão da importância da avaliação de desempenho para pequenas e médias empresas e tratandose de organizações com características próprias, alguns aspectos necessitam ser considerados, como a disponibilidade de recursos financeiros, a experiência dos gestores em lidar com o desempenho organizacional e com estratégias de longo prazo, e fatores internos e externos que interferem no negócio, demonstrando a necessidade da implementação de mecanismos que permitam o alinhamento entre o planejado e o realizado, a exemplo dos sistemas de avaliação de desempenho organizacional. 


\subsection{SISTEMAS DE AVALIAÇÃO DE DESEMPENHO ORGANIZACIONAL}

Os instrumentos de avaliação de desempenho fazem parte da história da humanidade desde os primórdios da civilização, haja vista que o ser humano, enquanto membro de determinado grupo social, é constantemente observado e avaliado por seus pares, e por isso, sempre se preocupou em avaliar suas atividades, corrigir erros e/ou melhorar ações. No ambiente empresarial essa característica também está presente por meio da avaliação de desempenho, complementando o processo gerencial de modo a garantir a continuidade e a competitividade empresarial (ALMEIDA; MARÇAL; KOVALESKI, 2004).

Nas últimas décadas, inúmeros pesquisadores dedicaram-se à criação de sistemas de avaliação de desempenho, os quais "estão alicerçados no pressuposto básico de que as empresas atuam em ambiente de recursos escassos e de alta complexidade, onde há enormes dificuldades em manter diferenciais competitivos a longo prazo" (SCHMIDT et al., 2006, p. 04). Na visão de Bortoluzzi et al. (2010, p. 07) os modelos com melhor embasamento e que melhor apresentam a formulação e implementação de estratégias, são:

QUADRO 01 - Modelos de sistemas de avaliação de desempenho geralmente encontrados na literatura

\begin{tabular}{|l|l|l|}
\hline \multicolumn{1}{|c|}{ FERRAMENTA } & \multicolumn{1}{|c|}{ AUTOR(ES) } & \multicolumn{1}{c|}{ DESCRIÇÃO } \\
\hline $\begin{array}{l}\text { Performance } \\
\text { Measurement Matrix }\end{array}$ & Keegan et al. (1989) & $\begin{array}{l}\text { Auxilia a empresa a definir seus objetivos estratégicos e } \\
\text { traduz esses objetivos em medida de desempenho. }\end{array}$ \\
\hline $\begin{array}{l}\text { Performance Pyramid } \\
\text { System }\end{array}$ & Lynch e Cross (1991) & $\begin{array}{l}\text { Evidencia as ligações entre a estratégia organizacional e } \\
\text { os objetivos operacionais. }\end{array}$ \\
\hline $\begin{array}{l}\text { Performance } \\
\text { for Service Industries }\end{array}$ & Fitzgerald et al. (1991) & $\begin{array}{l}\text { Baseia-se em seis dimensões que buscam ligar os } \\
\text { sistemas de ADO à estratégia e competitividade. }\end{array}$ \\
\hline $\begin{array}{l}\text { Balanced ScoreCard } \\
\text { Integrated } \\
\text { Performance } \\
\text { Measurement System }\end{array}$ & Kaplan e Norton (1992) & $\begin{array}{l}\text { É baseada em quatro perspectivas: financeira, clientes, } \\
\text { processos internos e aprendizado e crescimento, } \\
\text { interligando a estratégia com as atividades operacionais. }\end{array}$ \\
\hline $\begin{array}{l}\text { Bestaca dois aspectos na mensuração do desempenho: } \\
\text { integração das áreas de negócios e a implantação de } \\
\text { políticas e estratégias; baseia-se em quatro níveis: } \\
\text { corporativo, unidades de negócios, atividades e } \\
\text { processos. }\end{array}$ \\
\hline
\end{tabular}

Fonte: adaptado de Bortoluzzi et al. (2010)

Em razão das diferentes necessidades e premissas empresariais, Schmidt et al. (2006, p. 04) afirmam que "a identificação e a estruturação de modelos de sistema de avaliação de desempenho podem ser consideradas um problema clássico na área de gestão empresarial, tendo sido abordadas de diversas formas ao longo do tempo", pois um modelo apenas representa parte da realidade pela visão e pelo modo de quem deseja entendê-la, mudá-la e controlá-la, não havendo um modelo que possa ser considerado completo.

Nesse sentido, como não há um modelo que possa ser considerado completo, para que seja útil à organização é necessário que o mesmo seja ao menos balanceado, considerando os indicadores selecionados, as ferramentas administrativas, a cultura organizacional e o modo de atuação dos gestores (MÜLLER, 2003). Na concepção de Waggoner, Neely e Kennerley (1999) um sistema de avaliação de desempenho organizacional necessita ser projetado, gerenciado e analisado 
periodicamente para que produza os resultados esperados, demonstrando ser capaz de ajustar-se às mudanças competitivas, sendo os mesmos normalmente compostos pelos seguintes elementoschave: a) procedimentos para coleta e processamento de dados; b) ferramentas para disseminação de informações relacionadas aos dados coletados e processados; c) mecanismos de aprendizagem organizacional; d) processo de revisão para assegurar a representatividade e agilidade do sistema.

Os procedimentos de coleta e processamento de dados correspondem aos meios e aos modos com os quais as organizações mensuram e avaliam suas atividades. As ferramentas para disseminação de informações correspondem aos meios pelos quais os responsáveis pela avaliação de desempenho repassam os resultados desse processo. Os mecanismos de aprendizagem organizacional compreendem as ações a serem tomadas para melhorias futuras. 0 processo de revisão para assegurar a representatividade e agilidade do sistema refere-se ao processo de analisar se os indicadores e aspectos considerados atendem eficientemente as necessidades da empresa (WAGGONER; NEELY; KENNERLY, 1999). Independentemente do modelo implementado, o mesmo pode ser identificado por meio de elementos essenciais.

QUADRO 02 - Elementos essenciais dos sistemas de avaliação de desempenho organizacional

\begin{tabular}{|c|c|}
\hline Elementos e Características & Autor(es) \\
\hline Alinhamento estratégico (relação causa e efeito) & $\begin{array}{l}\text { Kaplan e Norton (2000); Yeo (2003); Garengo, Biazzo e } \\
\text { Bitici (2005); Punniyamoorthy e Murali (2008) }\end{array}$ \\
\hline Consideração de todos os níveis organizacionais & Yeo (2003) \\
\hline $\begin{array}{l}\text { Visão estratégica da organização } \\
\text { (perspectiva futura) }\end{array}$ & $\begin{array}{l}\text { Kaplan e Norton (2005); Tuomela (2005); Jacobsen, } \\
\text { Hofman-Bang e Nordby (2005); Garengo, Biazzo e Bitici } \\
\text { (2005); Punniyamoorthy e Murali (2008); Han (2004) }\end{array}$ \\
\hline Sistema de feedback (comunicação) & Tuomela (2005) \\
\hline Missão da organização & $\begin{array}{l}\text { Jacobsen, Hofman-Bang e Nordby (2005); } \\
\text { Punniyamoorthy e Murali (2008) }\end{array}$ \\
\hline Objetivos organizacionais & Skrinjar, Bosilj-Vuksic e Indihar-Stemberger (2008) \\
\hline Foco em stakeholders internos e externos & $\begin{array}{l}\text { Yeo (2003); Han (2004); Jacobsen, Hofman-Bang e Nordby } \\
\text { (2005); Garengo, Biazzo e Bitici (2005); Skrinjar, Bosilj- } \\
\text { Vuksic e Indihar-Stemberger (2008) }\end{array}$ \\
\hline Medidas financeiras e não financeiras & $\begin{array}{l}\text { Kaplan e Norton (2005); Yeo (2003); Tuomela (2005); } \\
\text { Garengo, Biazzo e Bitici (2005); Chenhall e Langfield-Smith } \\
\text { (2007); Skrinjar, Bosilj-Vuksic e Indihar-Stemberger } \\
\text { (2008); Punniyamoorthy e Murali (2008) }\end{array}$ \\
\hline
\end{tabular}

Fonte: adaptado de Bortoluzzi et al. (2010)

Os modelos de avaliação de desempenho possuem características intrínsecas e refletem a percepção de seus respectivos autores em relação ao adequado processo de avaliação de desempenho organizacional. Em razão dessa variedade, Müller (2003) afirma que a escolha entre um ou outro é feita em consonância com os objetivos da empresa e o grau de informação requerido, cabendo ao gestor escolher aquele que supra suas necessidades.

\subsubsection{INDICADORES DE DESEMPENHO}

Segundo Moreira (2007), há diferenças entre medida e indicador que necessitam ser analisadas para se compreender o que são: (a) a medida é um atributo qualitativo ou quantitativo destinado a avaliar algo por meio de comparação entre o realizado e o planejado; (b) o indicador corresponde ao resultado de determinar medida, tornando possível a compreensão daquilo que se pretende avaliar a partir dos limites estabelecidos. 
A definição de um conjunto de indicadores é necessária para o processo de avaliação de desempenho organizacional (FREZATTI et al., 2009), entretanto não é um tarefa fácil, pois a estruturação desse conjunto é uma complexa atividade que demanda a atribuição de valor em sentido qualitativo ou quantitativo, sendo uma das principais dificuldades a formulação e a parametrização do conjunto (SCHMIDT et al., 2006), razão pela qual é fundamental que a estrutura de indicadores utilizados em determinada organização seja coerente e que esteja alinhada com a estratégia organizacional (LUITZ; REBELATO, 2003).

Harrington (1993) apud Schmidt et al. (2006, p. 21) afirma que "os indicadores de desempenho são importantes na medida em que proporcionam o aperfeiçoamento do gerenciamento das organizações". Luitz e Rebelato (2003, p. 01), nesse sentido, afirmam que

\begin{abstract}
a estrutura para medição de desempenho é sempre baseada nos indicadores de desempenho, o que leva a concluir que se uma determinada organização não utiliza indicadores relevantes e coerentes, altamente relacionados aos seus pontos críticos de sucesso, com no mínimo um razoável índice de precisão, de nada adiantará determinar estratégias ou realizar benchmarking, simplesmente pelo fato de que nenhuma conclusão confiável a respeito de seu desempenho poderá ser formulada.
\end{abstract}

Em relação à análise dos indicadores, Silva e Niyama (2011, p. 217) afirmam que a mesma "necessita ser realizada de forma integrada, ou seja, análise conjunta de indicadores financeiros e não financeiros", permitindo que a alta administração acompanhe o desempenho da organização por meio de indicadores financeiros com foco em resultados, ou por meio de indicadores não financeiros com foco no processo de gestão. A literatura apresenta inúmeros indicadores, os quais apresentam variadas formulações e interpretações. As organizações necessitam tirar proveito dessa vasta gama de indicadores, haja vista ser fundamental que uma entidade disponha de diferentes indicadores, caso contrário, seu processo de avaliação de desempenho será limitado e focado em determinado aspecto sem a necessária abrangência; noutro norte, o excesso de indicadores pode fazer com que a organização perca seu foco (SCHMIDT et al., 2006). Nesse contexto, portanto, a escolha dos indicadores há de ser realizada pelos gestores consoante às necessidades da organização.

\title{
3 PROCEDIMENTOS METODOLÓGICOS
}

Esta pesquisa foi aplicada em concessionárias de máquinas e implementos agrícolas localizadas no município de Sinop/MT, cidade com relevante ascensão na região norte do Estado de Mato Grosso em razão do expressivo crescimento do agronegócio, com o objetivo de analisar como é o processo de avaliação de desempenho em pequenas e médias empresas, as quais recebem essa classificação com base em parâmetros objetivos, como a quantidade de empregados e o faturamento anual (PARISI; MEGLIORI, 2011). Na presente pesquisa, a classificação do porte empresarial seguiu os parâmetros do Instituto Brasileiro de Geografia e Estatística (IBGE, 2012), em que uma organização caracteriza-se como pequena quando possui entre 10 e 49 funcionários e como média quando possui entre 50 e 99 funcionários.

O presente estudo caracteriza-se como descritivo (SILVA, 2010), com a qual descreveu-se o processo de avaliação de desempenho em pequenas e médias empresas. Em relação aos procedimentos utilizados, caracteriza-se como um estudo multicaso (YIN, 2010), pois analisou o caso das concessionárias de máquinas e implementos agrícolas do município de Sinop/MT. Quanto à abordagem do problema, a presente pesquisa caracteriza-se como quali-quantitativa (SANTOS, 2009), sendo os dados tratados por meio de estatística descritiva, quantificados e qualificados para permitir a análise e interpretação dos resultados. 
Com a identificação das empresas que compõem a população das concessionárias de máquinas e implementos agrícolas, realizou-se um contato precedente para informar o foco e o delineamento do estudo e solicitar a participação na pesquisa. Entre as oito empresas que compõem essa população, três não demonstraram interesse em participar, sendo a pesquisa realizada, portanto, com as demais empresas, cuja amostra de participação corresponde a $62,50 \%$ da população. Com a definição das unidades de análise, discriminadas por meio de letras a fim de obstar a identificação e manter o anonimato, iniciou-se o processo de coleta de dados, realizado por meio de entrevistas semiestruturadas (MARTINS; THEÓPHILO, 2009).

O tratamento dos dados coletados foi realizado considerando-se os seguintes aspectos: i) caracterização das empresas e dos entrevistados; ii) caracterização do processo de avaliação de desempenho, com base nos elementos-chave descritos por Waggoner, Neely e Kennerley (1999), apresentados anteriormente. Ao final foi realizado um teste não paramétrico de hipóteses do tipo qui-quadrado (BISQUERRA; MARTINEZ; SARRIERA, 2004), sendo testadas três hipóteses: H1: há relação entre a formação do(s) gestor(es) e a realização da avaliação de desempenho; H2: há relação entre a correção ou aperfeiçoamento de ações e a realização da avaliação de desempenho; H3: a avaliação de desempenho é realizada e seus resultados são disseminados para todos os colaboradores em suas respectivas empresas.

\section{RESULTADOS}

\subsection{DESCRIÇÃO E ANÁLISE DOS DADOS}

A descrição do processo de avaliação de desempenho realizado nas concessionárias de máquinas e implementos agrícolas estudadas demandou a prévia caracterização das organizações e dos entrevistados, conforme demonstrado no Quadro 03.

Quadro 03: Caracterização das empresas e dos entrevistados

\begin{tabular}{|c|c|c|c|c|c|c|}
\hline & $\begin{array}{c}\text { Período em } \\
\text { Atividade }\end{array}$ & Colaboradores & $\begin{array}{l}\text { Faturamento } \\
\text { Médio Mensal } \\
\end{array}$ & Entrevistado & $\begin{array}{c}\text { Idade do } \\
\text { Entrevistado }\end{array}$ & $\begin{array}{c}\text { Qualificação Profissional } \\
\text { do Entrevistado }\end{array}$ \\
\hline A & $\begin{array}{l}\text { Há mais de } \\
\text { cinco anos }\end{array}$ & $\begin{array}{l}\text { Entre } 11 \text { e } 20 \\
\text { funcionários }\end{array}$ & $\begin{array}{c}\text { Acima de } \\
\mathrm{R} \$ 500.000,00\end{array}$ & Gestor & $\begin{array}{l}\text { Acima de } 55 \\
\text { anos }\end{array}$ & $\begin{array}{c}\text { Curso de } \\
\text { aperfeiçoamento em } \\
\text { vendas e gestão }\end{array}$ \\
\hline B & $\begin{array}{l}\text { Há mais de } \\
\text { cinco anos }\end{array}$ & $\begin{array}{l}\text { Entre } 11 \text { e } 20 \\
\text { funcionários }\end{array}$ & $\begin{array}{c}\text { Acima de } \\
\mathrm{R} \$ 500.000,00\end{array}$ & Gestor & $\begin{array}{c}\text { Entre } 26 \text { e } 35 \\
\text { anos }\end{array}$ & $\begin{array}{c}\text { Curso de } \\
\text { aperfeiçoamento em } \\
\text { vendas e gestão }\end{array}$ \\
\hline C & $\begin{array}{l}\text { Há mais de } \\
\text { cinco anos }\end{array}$ & $\begin{array}{l}\text { Entre } 21 \text { e } 30 \\
\text { funcionários }\end{array}$ & $\begin{array}{c}\text { Acima de } \\
\mathrm{R} \$ 500.000,00\end{array}$ & Gestor & $\begin{array}{c}\text { Entre } 36 \text { e } 45 \\
\text { anos }\end{array}$ & $\begin{array}{c}\text { Curso de } \\
\text { aperfeiçoamento em } \\
\text { vendas e gestão }\end{array}$ \\
\hline D & $\begin{array}{l}\text { Há mais de } \\
\text { cinco anos }\end{array}$ & $\begin{array}{l}\text { Entre } 31 \text { e } 40 \\
\text { funcionários }\end{array}$ & $\begin{array}{c}\text { Acima de } \\
\mathrm{R} \$ 500.000,00\end{array}$ & Gestor & $\begin{array}{c}\text { Entre } 36 \text { e } 45 \\
\text { anos }\end{array}$ & $\begin{array}{c}\text { Curso superior voltado } \\
\text { à gestão }\end{array}$ \\
\hline$E$ & $\begin{array}{l}\text { Há mais de } \\
\text { cinco anos }\end{array}$ & $\begin{array}{l}\text { Entre } 21 \text { e } 30 \\
\text { funcionários }\end{array}$ & $\begin{array}{c}\text { Acima de } \\
R \$ 500.000,00\end{array}$ & Gestor & $\begin{array}{c}\text { Acima de } 55 \\
\text { anos }\end{array}$ & $\begin{array}{c}\text { Curso superior voltado } \\
\text { à gestão }\end{array}$ \\
\hline
\end{tabular}

Fonte: dados da pesquisa (2013)

Com base nas informações apresentadas no Quadro 03, essas organizações caracterizam-se como de pequeno porte, atuam há mais de cinco anos no setor de máquinas e implementos agrícolas com faturamento médio mensal superior a $\mathrm{R} \$ 500.000,00$. As informações foram obtidas por meio de entrevistas com os gestores dessas organizações, os quais possuem as seguintes características: ambos são do sexo masculino; possuem pelo menos um curso de aperfeiçoamento voltado à área de gestão e vendas, entretanto, apenas 02 possuem cursos superiores com foco em gestão. Em relação 
à atuação nesse mercado, três atuam entre 01 e 05 anos, um atua entre 11 e 15 anos e os demais atuam há mais de 15 anos.

Constatou-se que dentre os agentes responsáveis pela gestão dessas organizações encontram-se as figuras de sócios e/ou proprietários e de gestores contratados, não sendo detectada a presença de um contador ou de um consultor externo ou de qualquer outro agente. Em todas as empresas estudadas os gestores realizam o planejamento das atividades, sendo que em quatro dessas organizações esse planejamento é realizado formalmente, enquanto que em uma é realizado informalmente, sendo tal planejamento realizado principalmente com base nos resultados anteriormente obtidos e na previsão de vendas para os próximos períodos, englobando os três níveis de controle (estratégico, tático e operacional).

No entanto, além do planejamento das atividades organizacionais, faz-se necessária a revisão desse planejamento de modo a possibilitar a análise das estratégias empresariais em relação ao desempenho tático e operacional. A frequência desse processo de revisão não é uniforme, conforme informações obtidas, ocorrendo mensalmente em $60 \%$ dessas empresas, trimestralmente em $20 \%$ delas, e anualmente em $20 \%$ nas demais organizações.

\subsection{AVALIAÇÃO DE DESEMPENHO EM PEQUENAS E MÉDIAS EMPRESAS}

O presente estudo descreve o processo de avaliação de desempenho em pequenas e médias empresas do ramo de concessionárias de máquinas e implementos agrícolas do município de Sinop/MT. Inicialmente foram identificadas as organizações que realizam ou não a avaliação de desempenho e, em relação àquelas que realizam, se o fazem formal ou informalmente, conforme demonstrado na Tabela 01.

TABELA 01 - Empresas que realizam o processo avaliação de desempenho

\begin{tabular}{|c|c|c|c|c|c|c|}
\hline \multirow{2}{*}{ DESCRIÇÃO } & \multicolumn{5}{|c|}{ EMPRESAS } & \multirow{2}{*}{ FREQUÊNCIA (\%) } \\
\hline & $A$ & B & $\mathrm{C}$ & $\mathrm{D}$ & $E$ & \\
\hline Sim, formalmente & $x$ & & $x$ & $\mathrm{x}$ & $x$ & $80,00 \%$ \\
\hline Sim, informalmente & & $x$ & & & & $20,00 \%$ \\
\hline Não & & & & & & $0,00 \%$ \\
\hline
\end{tabular}

Fonte: dados da pesquisa (2013)

Embora os gestores de todas as empresas estudadas afirmarem que realizam a avaliação de desempenho, constatou-se que quatro a fazem formalmente e um informalmente. No entanto, o gestor da Empresa B afirmou que o planejamento realizado na empresa não contempla "metas e objetivos", razão pela qual não é possível a realização da avaliação de desempenho, pois não apresenta um dos elementos essenciais desse processo (conforme descrição do Quadro 02), impossibilitando a comparação entre o planejado e o realizado. Nesse contexto, a caracterização da avaliação de desempenho em pequenas e médias empresas foi realizada considerando-se apenas as empresas que contemplam os elementos essenciais para essa finalidade. Na sequência, foram descritos os processos de avaliação de desempenho realizados, nessas empresas, com base nos elementos-chave descritos por Waggoner, Neely e Kennerley (1999), caracterizados a seguir.

\subsubsection{PROCEDIMENTOS PARA COLETA E PROCESSAMENTO DE DADOS}

Segundo os gestores, o processo de avaliação de desempenho realiza-se com base nos dados descritos em relatórios contábeis, relatórios financeiros, relatórios de compras e vendas, relatórios 
de satisfação de clientes e relatórios de satisfação e desempenho de seus colaboradores. Em nenhuma das empresas foi implementado algum sistema de avaliação de desempenho abordado na literatura, segundo os gestores em razão do desconhecimento da existência dessas ferramentas e/ou dos benefícios por elas gerados.

\section{(a) Relatórios Contábeis, Relatórios Financeiros, Relatórios de Compras e Vendas}

Os relatórios contábeis, os relatórios financeiros e relatórios de compra e venda são gerados por meio de softwares de automação comercial, os quais têm por finalidade registrar entradas e saídas de numerários e de mercadorias; fornecer informações ao processo decisório; auxiliar no controle interno e no desenvolvimento organizacional.

\section{(b) Relatório de Satisfação dos Clientes}

A satisfação de clientes refere-se à satisfação com as marcas representadas, motivo que faz com que as concessionárias: (a) trabalhem com produtos que melhor supram as necessidades de seus clientes; (b) estejam constantemente atentas e atualizadas em relação aos produtos e/ou serviços disponibilizados; e (c) ofereçam serviços de pós-venda, inclusive com acompanhamento e treinamento em novas máquinas e/ou implementos.

\section{(c) Satisfação e Desempenho dos Colaboradores}

A satisfação e o desempenho de colaboradores são mensurados e avaliados por processos e critérios de competência, pelos quais analisa-se o cumprimento ou não das metas e dos objetivos previamente determinados, bem como os motivos do cumprimento ou não, e os mecanismos necessários ao bom e fiel cumprimento. Outro meio de mensurar e avaliar a satisfação e o desempenho dos colaboradores é permitindo o acesso destes à alta administração, possibilitando a cada colaborador relatar sua satisfação ou insatisfação para com a empresa, bem como sugerir meios a serem implementados para satisfazê-lo.

Um relevante aspecto a ser considerado no processo de avaliação de desempenho são os indicadores utilizados, em relação aos quais os gestores foram unânimes em afirmar que utilizam indicadores financeiros e não financeiros, conforme explicitado nas Tabelas 02 e 03.

TABELA 02 - Indicadores financeiros utilizados no processo de avaliação de desempenho organizacional

\begin{tabular}{l|c|c|c|c|c|c|c}
\hline \multirow{2}{*}{ DESCRIÇÃO } & \multicolumn{5}{|c|}{ EMPRESAS } & \multirow{2}{*}{ FREQUÊNCIA (\%) } \\
\cline { 2 - 6 } & $\mathrm{A}$ & $\mathrm{B}$ & $\mathrm{C}$ & $\mathrm{D}$ & $\mathrm{E}$ & $100,00 \%$ \\
\hline Rentabilidade & $\mathrm{x}$ & - & $\mathrm{x}$ & $\mathrm{x}$ & $\mathrm{x}$ & $100,00 \%$ \\
\hline Liquidez & $\mathrm{x}$ & - & $\mathrm{x}$ & $\mathrm{x}$ & $\mathrm{x}$ & $100,00 \%$ \\
\hline Faturamento & $\mathrm{x}$ & - & $\mathrm{x}$ & $\mathrm{x}$ & $\mathrm{x}$ & $75,00 \%$ \\
\hline Gastos & $\mathrm{x}$ & - & $\mathrm{x}$ & & $\mathrm{x}$ & $0,00 \%$ \\
\hline Outro(s) & & - & & & & \\
\hline
\end{tabular}

Fonte: dados da pesquisa (2013)

TABELA 03 - Indicadores não financeiros utilizados na avaliação de desempenho organizacional

\begin{tabular}{|c|c|c|c|c|c|c|}
\hline \multirow{2}{*}{ DESCRIÇÃOO } & \multicolumn{5}{|c|}{ EMPRESAS } & \multirow{2}{*}{ FREQUÊNCIA (\%) } \\
\hline & A & $\mathrm{B}$ & $\mathrm{C}$ & $\mathrm{D}$ & $\mathrm{E}$ & \\
\hline Participação no Mercado & $x$ & - & $x$ & $x$ & $x$ & $100,00 \%$ \\
\hline Ações Socioambientais & & - & & & $x$ & $25,00 \%$ \\
\hline Perspectivas e Satisfação de Clientes & $x$ & - & $x$ & $x$ & $\mathrm{x}$ & $100,00 \%$ \\
\hline
\end{tabular}




\begin{tabular}{l|c|c|c|c|c|c|c} 
Satisfação de Colaboradores & $\mathrm{x}$ & - & $\mathrm{x}$ & $\mathrm{x}$ & $\mathrm{x}$ & $100,00 \%$ \\
\hline Rotatividade de Estoque & $\mathrm{x}$ & - & $\mathrm{x}$ & $\mathrm{x}$ & $\mathrm{x}$ & $100,00 \%$ \\
\hline Outro(s) & & - & & & & $0,00 \%$ \\
\hline
\end{tabular}

Fonte: dados da pesquisa (2013)

Em relação à periodicidade, a avaliação de desempenho é realizada mensalmente e trimestralmente, na proporção demonstrada na Tabela 04, cujos períodos de tempo são, segundo os gestores, suficientes para atender as necessidades das empresas.

TABELA 04 - Frequência de realização do processo de avaliação de desempenho organizacional

\begin{tabular}{l|c|c|c|c|c|c|c}
\hline \multicolumn{1}{c}{ DESCRIÇÃO } & \multicolumn{6}{c|}{ EMPRESAS } & \multirow{2}{*}{ FREQUÊNCIA (\%) } \\
\cline { 2 - 6 } & $\mathrm{A}$ & $\mathrm{B}$ & $\mathrm{C}$ & $\mathrm{D}$ & $\mathrm{E}$ & $50,00 \%$ \\
\hline Mensalmente & & - & $\mathrm{x}$ & & $\mathrm{x}$ & $50,00 \%$ \\
\hline Trimestralmente & $\mathrm{x}$ & - & & $\mathrm{x}$ & & $0,00 \%$ \\
\hline Semestralmente & & - & & & & $0,00 \%$ \\
\hline Anualmente & & - & & & & $0,00 \%$ \\
\hline Esporadicamente & & - & & & & \\
\hline
\end{tabular}

Fonte: dados da pesquisa (2013)

Entre os agentes responsáveis pelo processo de avaliação de desempenho nessas organizações encontram-se os sócios e/ou proprietários e os gestores, ou seja, os mesmos agentes responsáveis pela gestão das organizações. A presença de um contador ou de um consultor externo ou de qualquer outro agente não foi detectada nesse processo.

\subsubsection{FERRAMENTAS PARA DISSEMINAÇÃO DE INFORMAÇÕES}

A integração entre os aspectos considerados e a forma como são coletados e processados constitui o primeiro elemento-chave, apresentado anteriormente. No entanto, faz-se necessário que as informações resultantes dessa integração sejam disseminadas entre seus interessados. Dessa forma, concomitantemente à realização da avaliação de desempenho são realizadas reuniões para analisar os resultados desse processo, com a participação dos gestores, dos sócios-proprietários e chefes de departamento.

Os resultados dessas reuniões são repassados aos demais colaboradores pelos gestores de cada unidade, para juntos avaliarem o desempenho, comparando o planejado com o realizado e os fatores que contribuíram ou não para a obtenção daqueles resultados. Essas informações são repassadas por meio de relatórios elaborados pelos responsáveis pelo processo de avaliação de desempenho, nos quais constam as metas e objetivos atingidos até aquele momento e aqueles a serem cumpridos em determinado prazo, as razões do cumprimento ou não cumprimento dessas metas e objetivos, e os mecanismos a serem empregados caso haja a necessidade de se corrigir ou implementar ações.

\subsubsection{MECANISMOS DE APRENDIZAGEM ORGANIZACIONAL}

Os mecanismos de aprendizagem organizacional relacionam-se às informações do processo de avaliação de desempenho, resultando em mudanças no modelo de gestão dessas organizações, apresentando-se como um sistema de feedback pelo qual os gestores conhecem as necessidades empresariais e, considerando a oscilação do agronegócio em razão de fatores externos às organizações, como clima, exigências de mercado, embargos às exportações, entre outros. Esses mecanismos permitem a implementação de ações para amenizar possíveis impactos que possam interferir no desempenho organizacional. 
Entre esses mecanismos destacam-se as consultorias sobre a perspectiva de mercado, programas de aperfeiçoamentos em gestão e de venda e pós-venda, a contratação de profissionais que já atuaram na área, e a implementação de softwares de gestão integrada. Ao final de cada exercício social, os responsáveis pela gestão e pelo processo de avaliação de desempenho reúnem-se novamente, desta vez para analisar se as ações implementadas resultaram ou não em melhorias para a organização e, partir de então, avaliar o desempenho referente àquele período e realizar o planejamento organizacional para períodos futuros.

\subsubsection{REVISÃO PARA ASSEGURAR A REPRESENTATIVIDADE E A AGILIDADE DO SISTEMA}

A revisão do sistema é realizada ao final de cada ano, observando-se consequentemente cumprimento ou não do planejamento empresarial para aquele período. Esse procedimento permite aos gestores identificarem as áreas que necessitam de monitoramento, principalmente no que tange ao processo de venda e de pós-venda, ao processo de comunicação e ao desempenho dos colaboradores sob sua responsabilidade.

A efetividade de um aspecto e/ou indicador utilizado na avaliação de desempenho realiza-se com a análise de uma ação melhorada ou implementada, pela qual os responsáveis pelo processo de avaliação de desempenho analisam se os meios para cumprir o planejamento foram ou não suficientes. Caso constatado que um desses aspectos não está auxiliando na correção ou implementação de ações que contribuam para o cumprimento do planejamento, há a imediata substituição por outro aspecto que os gestores julgam aptos a desempenhar essa finalidade. Se constatado que os aspectos estão contribuindo com o cumprimento do planejamento, os gestores os mantêm, assegurando a representatividade e a agilidade do sistema de avaliação de desempenho, pelo menos por mais um período.

Nesse contexto, percebe-se a importância do processo de avaliação de desempenho para o sucesso empresarial, contribuindo significativamente para o aperfeiçoamento da gestão e para o cumprimento das metas e objetivos contemplados no planejamento organizacional.

\subsection{TESTES DE HIPÓTESES}

Nesta etapa da pesquisa apresentam-se os testes de hipóteses realizados por meio do cruzamento de variáveis e a respectiva análise da relação existente com base no grau de significância estatística correspondente a 0,05 (BISQUERRA; MARTINEZ; SARRIERA, 2004),para os quais se utilizou da metodologia de estatística descritiva do tipo qui-quadrado, testando-se as seguintes hipóteses: $\mathrm{H} 1$ : há relação entre a formação dos gestores e a realização da avaliação de desempenho; H2: há relação entre a correção ou aperfeiçoamento de ações e a realização da avaliação de desempenho; H3: a avaliação de desempenho é realizada e os resultados são disseminados para todos os colaboradores em suas respectivas empresas.

\subsubsection{HIPÓTESE 1}

$\checkmark \quad$ H1: há relação entre a formação dos gestores e a avaliação de desempenho. 
O teste desta hipótese visou comprovar se há relação entre a realização da avaliação de desempenho e o nível de formação do(s) gestor(es) das organizações. Conforme os resultados demonstrados na Tabela 05 a presente hipótese possui um p-valor de 0,361.

TABELA 05 - Formação do gestor x realização da avaliação de desempenho

\begin{tabular}{l|c|c|c}
\hline \multirow{2}{*}{ FORMAÇÃO DO GESTOR } & REALIZA AVALIAÇÃO DE DESEMPENHO & \multirow{2}{*}{ TOTAL } \\
\cline { 2 - 4 } & SIM & NÃO & 2 \\
\hline Nível Superior & 2 & 1 & $40 \%$ \\
Técnico/Profissionalizante & 2 & 1 & $60 \%$ \\
\hline TOTAL & 4 & & $100 \%$ \\
\hline Teste Qui-Quadrado & & & \\
Nível $\alpha$ de Significância $=0,361$ & & & \\
\hline
\end{tabular}

Fonte: dados da pesquisa (2013)

O resultado desta hipótese demonstra que o valor do teste qui-quadrado possui relevância estatística maior que 0,05. Desse modo, rejeita-se a hipótese testada, inferindo-se que não há relação entre a formação dos gestores e a realização da avaliação de desempenho, sendo tal processo realizado mesmo que o gestor não possua formação na área de gestão.

\subsubsection{HIPÓTESE 2}

H2: há relação entre a correção de ações e a realização do processo de avaliação

O teste da presente hipótese visou comprovar a relação entre a correção ou aperfeiçoamento de ações e a realização da avaliação de desempenho. Os resultados demonstrados na Tabela 06 demonstram que a presente hipótese possui um p-valor de 0,025.

TABELA 06 - Realização da avaliação de desempenho x correção e/ou aperfeiçoamento de ações

\begin{tabular}{l|c|c|c}
\hline \multirow{2}{*}{ INDICADORES } & REALIZA AVALIAÇÃO DE DESEMPENHO & \multirow{2}{*}{ TOTAL } \\
\cline { 2 - 4 } & SIM & NÃO & \\
\hline Corrige e/ou aperfeiçoa ações & 4 & 0 & $80 \%$ \\
\hline Não corrige e/ou aperfeiçoa ações & 0 & 1 & 1 \\
\hline TOTAL & 4 & 1 & $20 \%$ \\
\hline
\end{tabular}

Teste Qui-Quadrado

Nível $\alpha$ de Significância $=0,025$

Fonte: dados da pesquisa (2013)

O resultado demonstra que o valor do teste qui-quadrado possui relevância estatística menor que 0,05, inferindo-se que há relação entre a correção ou o aperfeiçoamento de ações e a realização da avaliação de desempenho, de modo que os resultados do processo de avaliação de desempenho de fato são utilizados no processo gerencial da empresa.

\subsubsection{HIPÓTESE 3}

H3: a avaliação de desempenho é realizada e seus resultados são disseminados para todos os colaboradores em suas respectivas empresas.

O teste desta hipótese visou comprovar a relação entre a realização da avaliação de desempenho e a disseminação dos resultados desse processo entre todos os colaboradores em suas respectivas empresas, cujos resultados demonstram um p-valor de 0,025 . 
TABELA 07 - Realização da avaliação de desempenho x disseminação de resultados

\begin{tabular}{|c|c|c|c|}
\hline \multirow{2}{*}{ DISSEMINAÇÃO DE RESULTADOS } & \multicolumn{2}{|c|}{ REALIZA AVALIAÇÃO DE DESEMPENHO } & \multirow{2}{*}{ TOTAL } \\
\hline & $\mathrm{SIM}$ & NÃO & \\
\hline Dissemina resultados para os colaboradores & 4 & 0 & $4 \quad 80 \%$ \\
\hline Não dissemina resultados para os colaboradores & 0 & 1 & $120 \%$ \\
\hline TOTAL & 4 & 1 & $5 \quad 100 \%$ \\
\hline $\begin{array}{l}\text { Teste Qui-Quadrado } \\
\text { Nível } \alpha \text { de Significância }=0,025\end{array}$ & & & \\
\hline
\end{tabular}

Fonte: dados da pesquisa (2013)

Com base no resultado apresentado na Tabela 07 aceita-se a hipótese testada, demonstrando que a avaliação de desempenho é realizada e seus resultados são disseminados para todos os colaboradores em suas respectivas empresas. Essa premissa é fundamental para que as ações de melhoria e aperfeiçoamento sejam implementadas. Desse modo, percebe-se que de fato as empresas estudadas, mesmo sem ter implantado algum dos sistemas de avaliação de desempenho mais recorrentes na literatura, empenham-se na busca da melhoria contínua no processo de gestão e, consequentemente, nos resultados obtidos pela organização.

Os testes de hipóteses permitiram demonstrar que as organizações efetivamente realizam o processo de avaliação de desempenho, cujos resultados demonstram o atendimento de premissas básicas desse processo, como, por exemplo, a disseminação dos resultados e o aperfeiçoamento e a correção de ações, demonstrando a presença de elementos-chave do processo de avaliação de desempenho descritos por Waggoner, Neely, Kennerley (1999).

\section{CONSIDERAÇÕES FINAIS E SUGESTÕES PARA FUTUROS TRABALHOS}

O presente estudo objetivou analisar o processo de avaliação de desempenho realizado em concessionárias de máquinas e implementos agrícolas, tendo como população as oito concessionárias de máquinas e implementos agrícolas, dentre as quais foi selecionada uma amostra de cinco organizações $(62,50 \%)$. Com a análise dos dados coletados constatou-se que as empresas compreendidas nesse estudo, com exceção de uma unidade, embora não façam uso de nenhum sistema de avaliação de desempenho abordado na literatura gerencial, realizam o processo de avaliação de desempenho, cumprindo todas as suas etapas, atendendo aos elementos-chave descritos por Waggoner, Neely e Kennerley (1999).

Os resultados evidenciados demonstram que os objetivos propostos foram cumpridos com a análise e descrição do processo de avaliação de desempenho organizacional, com a descrição de como as organizações avaliam seus desempenhos e quais os indicadores, além dos aspectos considerados nesse processo. Ressalta-se que nenhuma das organizações analisadas implementou algum modelo de avaliação de desempenho abordado na literatura.

Com a realização dessa pesquisa constatou-se que o processo de avaliação de desempenho é útil e necessária também às empresas pequenas e médias empresas, assegurando o cumprimento do planejamento organizacional, subsidiando o controle gerencial das concessionárias de máquinas e implementos agrícolas do município de Sinop/MT, com resultados fundamentais para o cumprimento das metas e objetivos, possibilitando a arguição da relação de causa e efeito do desempenho organizacional, fornecendo informações úteis e tempestivas, facilitando a análise crítica do desempenho global da empresa e permitindo a relação entre o desempenho passado, presente e esperado para futuro da organização. Quanto aos indicadores, constatou-se a utilização de 
indicadores financeiros e não financeiros, como rentabilidade, liquidez, faturamento, participação no mercado e ações socioambientais.

Conclui-se que o processo de avaliação de desempenho é útil e necessário às empresas, inclusive para as organizações de pequeno e médio porte, pois assegura o planejamento organizacional, supre necessidades gerenciais e auxilia na tomada de decisões. Cabe ressaltar que as informações apresentadas refletem as opiniões dos entrevistados. Em função de limitações na realização deste estudo, como a limitação geográfica e a restrição às concessionárias de máquinas e implementos agrícolas, sugere-se a realização de novas pesquisas em outras empresas de pequeno e médio porte, com aspectos não apresentados neste trabalho, como os métodos e procedimentos empregados na avaliação de desempenhos.

\section{REFERÊNCIAS}

ALMEIDA, S.; MARÇAL, R. F. M.; KOVALESKI, J. L. Metodologias para avaliação de desempenho organizacional. In: Encontro Nacional de Engenharia de Produção, 24, 2004, Florianópolis. Anais do XXIV ENEGEP, Florianópolis: ABEPRO, 2004.

BISQUERRA, R.; MARTINEZ, F.; SARRIERA, J. C. Introdução à estatística: enfoque informático com o pacato estatístico SPSS. 1. ed. Porto Alegre: Artmed, 2004.

BORTOLUZZI, S. C.; ENSSLIN, S. R.; ENSSLIN, L. Práticas de avaliação de desempenho organizacional em pequenas e médias empresas: investigação em uma empresa de porte médio do ramo moveleiro. Revista Produção Online, v. 10, n. 03, p. 551-557, 2010.

CORRÊA, H. L.; JUNIOR, F. H. Sistemas de mensuração e avaliação de desempenho organizacional: estudo de casos no setor químico. Revista Contabilidade e Finanças, v. 19, n. 48, p. 50-64, set./dez. 2008.

DUTRA, A. Metodologias para avaliar o desempenho organizacional: revisão e proposta de uma abordagem multicritério. Revista Contemporânea de Contabilidade, ano 2, v. 1, p. 25-56, 2005.

FREZATTI, F.; ROCHA, W.; NASCIMENTO, A. R.; JUNQUEIRA, E. Controle gerencial: uma abordagem da contabilidade gerencial no contexto econômico, comportamental e sociológico. São Paulo: Atlas, 2009.

GARENGO, P.; BIAZZO, S.; BITITCI, U. S. Performance measurement systems in SMEs: a review for a research agenda. International Journal of Management Reviews, v. 7, n. 1, p. 25-47, 2005.

IBGE, Instituto Brasileiro de Geografia e Estatística. Micros e pequenas empresas comerciais e de serviços no Brasil. Disponível em

<http://www.ibge.gov.br/home/estatistica/economia/microempresa>. Acesso em 27 jul. 2012.

LEONE, N. M. C. P. G. As especificidades das pequenas e médias empresas. Revista de Administração da Universidade de São Paulo, v. 34, n. 02, p. 91-94, abr./jun. 1999.

LUITZ, M. P.; REBELATO, M. G. Avaliação de desempenho organizacional. In: Encontro Nacional de Engenharia de Produção, 23, 2003, Ouro Preto. Anais do XXIII ENEGEP, Ouro Preto: ABREPRO, 2003.

MARTINS, G. A.; THEÓPHILO, C. R. Metodologia da investigação científica para ciências sociais aplicadas. 2. ed. São Paulo: Atlas, 2009. 
MORAES, G. D. A.; TERENCE, A. C. F.; BIGATON, A. L. W.; ESCRIVÃO FILHO, E. A estruturação organizacional das micro e pequenas empresas. In: Simpósio de Excelência em Gestão e Tecnologia, 3, 2007, Resende. Anais do III SEGET... Resende: AEDB, 2007.

MOREIRA, E. Proposta de uma sistemática para o alinhamento das ações operacionais aos objetivos estratégicos, em uma gestão orientada por indicadores de desempenho, 189 f., 2007. Tese (Doutorado em Engenharia de Produção). Universidade Federal de Santa Catarina, Florianópolis.

MÜLLER, C. J. Modelo de gestão integrando planejamento estratégico, sistemas de avaliação de desempenho e gerenciamento de processos, 253 f., 2003. Tese (Doutorado em Engenharia de Produção). Universidade Federal de Rio Grande do Sul, Porto Alegre.

NEELY, A. D; MILLS, J.; PLATTS, K.; RICHARDS, H.; GREGORY, M.; BOURNE, M.; KENNERLEY, M. P. Performance measurement system design: developing and testing a process-based approach. International Journal of Operations \& Production Management, v. 20, n. 10, p. 1119-1145, 2000.

PARISI, C.; MEGLIORINI, E. Contabilidade Gerencial. São Paulo: Atlas, 2011.

SANTOS, R. V. Controladoria: uma introdução ao sistema de gestão econômica. São Paulo: Saraiva, 2010.

SANTOS, T. S. Do artesanato intelectual ao contexto virtual: ferramentas metodológicas para a pesquisa social. Sociologias, Porto Alegre, ano 11, n. 21, p. 120-156, jan./jun. 2009.

SCHMIDT, P.; SANTOS, J. L.; MARTINS, M. A. Avaliação de empresas: foco na análise de desempenho para o usuário interno (teoria e prática). São Paulo: Atlas, 2006.

SCHMIDT, P.; SANTOS, J. L.; PINHEIRO P. R. Introdução à contabilidade gerencial. Coleção Resumos de Contabilidades. São Paulo: Atlas, 2007.

SILVA, A. C. R. Metodologia da pesquisa aplicada à contabilidade. São Paulo: Atlas, 2010.

SILVA, C. A. T.; NIYAMA, J. K. (Org.). Contabilidade para concursos e exame de suficiência. São Paulo: Atlas, 2011.

SOUZA, A. E. Indicadores de mensuração de desempenho em pequenas e médias empresas: estudo no setor calçadista de Santa Catarina, 248 f., 2011. Tese (Doutorado em Administração). Faculdade de Economia, Administração e Ciências Contábeis, Universidade de São Paulo, São Paulo.

WAGGONER, D. B.; NEELY, A. D.; KENNERLEY, M. P. The forces that shape organizational performance measurement system: an interdisciplinary review. International Journal of Production Economics, v. 60, p. 53-60, abr. 1999.

YIN, R. K. Estudo de caso: planejamento e métodos. 4. ed. Porto Alegre: Bookman, 2010. 